
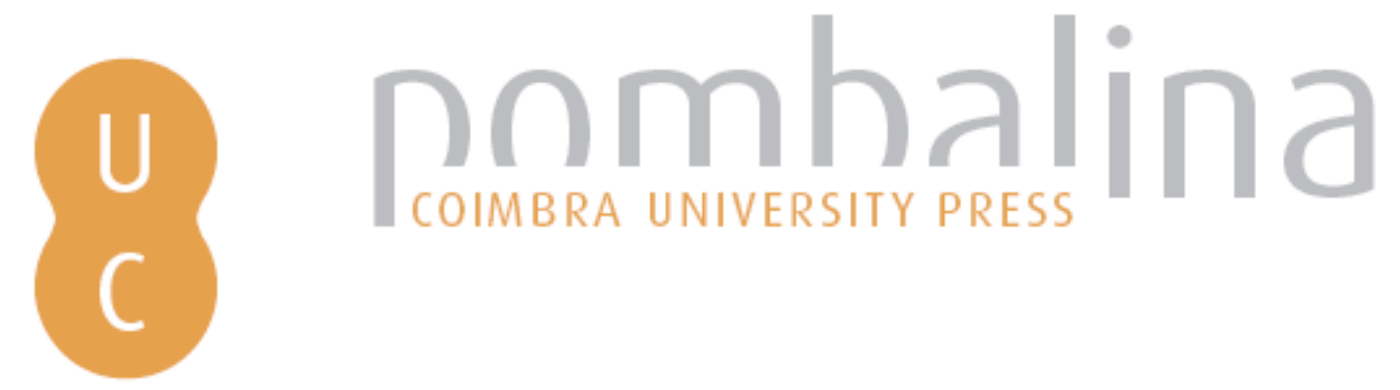

\title{
AdriaFirePropagator and AdriaFireRisk: user friendly Web based wildfire propagation and wildfire risk prediction software
}

Autor(es): $\quad$ Bugari, Marin; Stipaniev, Darko; Jakovevi, Toni

Publicado por: Imprensa da Universidade de Coimbra

URL

persistente: URI:http://hdl.handle.net/10316.2/44615

DOI: $\quad$ DOI:https://doi.org/10.14195/978-989-26-16-506_98

Accessed : $\quad$ 26-Apr-2023 13:49:49

A navegação consulta e descarregamento dos títulos inseridos nas Bibliotecas Digitais UC Digitalis, UC Pombalina e UC Impactum, pressupõem a aceitação plena e sem reservas dos Termos e Condições de Uso destas Bibliotecas Digitais, disponíveis em https://digitalis.uc.pt/pt-pt/termos.

Conforme exposto nos referidos Termos e Condições de Uso, o descarregamento de títulos de acesso restrito requer uma licença válida de autorização devendo o utilizador aceder ao(s) documento(s) a partir de um endereço de IP da instituição detentora da supramencionada licença.

Ao utilizador é apenas permitido o descarregamento para uso pessoal, pelo que o emprego do(s) título(s) descarregado(s) para outro fim, designadamente comercial, carece de autorização do respetivo autor ou editor da obra.

Na medida em que todas as obras da UC Digitalis se encontram protegidas pelo Código do Direito de Autor e Direitos Conexos e demais legislação aplicável, toda a cópia, parcial ou total, deste documento, nos casos em que é legalmente admitida, deverá conter ou fazer-se acompanhar por este aviso. 


\section{ADVANCES IN}

\section{FOREST FIRE RESEARCH}

\section{8}

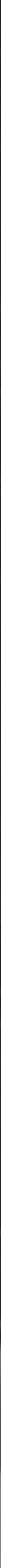




\title{
AdriaFirePropagator and AdriaFireRisk - user friendly Web based wildfire propagation and wildfire risk prediction software
}

\author{
Marin Bugarić*; Darko Stipaničev; Toni Jakovčević \\ ${ }^{1}$ University of Split Faculty of Electrical Engineering, Mechanical Engineering and Naval \\ Architecture in Split, Ruđera Boškovića 32, 21000 Split, Croatia, \{marin.bugaric@fesb.hr*, \\ darko.stipanicev@fesb.hr,toni.jakovcevic@fesb.hr\}
}

\begin{abstract}
The HOLISTIC Project with full name Adriatic Holistic Forest Fire Protection was a project funded by Adriatic IPA funds that has gathered together almost all regions around Adriatic Sea in order to improve wildfire prevention, protection, fighting and post-fire recuperation. It has started in December 2013 and ended in December 2016. A part of this project was to establish user friendly and simple regional wildfire propagation and wildfire risk prediction simulation tools that could be easily used by fire fighters in everyday fire fighting operative work, but also as a tool for fire season preparation. The final results were AdriaFirePropagator and AdriaFireRisk and additionally AdriaFireRisk sub-module named AdriaFireRiskPanels, and they are the main topics of this paper. AdriaFirePropagator is a Web based system for wildfire behaviour modelling and wildfire spread simulation. Web based means that all the data are stored on the server, and all the applications in the fire simulation software are server based programs that run when requested by the users and the only user interface is a standard web browser. AdriaFireRisk is additional module, running parallel on the same server using the same GIS calculation engine. AdriaFireRisk calculates site-specific wildfire risk index based on experimental system for micro location wildfire risk index calculation developed for Split - Dalmatia County. AdriaFireRisk is also a support system for additional HOLISTIC project result - AdriaFireRiskPanels designed to raise the awareness about the wildfire risk in order to increase caution and reduce the number of accidental wildfires. The paper describes technical details of these systems, as well as their practical implementation.
\end{abstract}

Keywords: wildfire propagation simulation, wildfire risk prediction, wildfires, forest fires, EU project

\section{Introduction}

The regions around Adriatic Sea have high risk of wildfires, especially during the summer due to very warm climate with low level of humidity, densely spaced Mediterranean vegetation, particularly conifer forests and lot of tourists. During fire season that is usually between July 1 and October 31, there are lot of wildfires in this region. For example in Republic Croatia located on the east side of Adriatic Sea in the last 10 years 2.320 wildfires have been recoded that affected 84.250 hectares of forests and forest land.

Therefore, the biggest Croatian county Split-Dalmatia County has initiated in 2007 preparation of strategic project target to Adriatic IPA funds, based on Split-Dalmatia County holistic approach to forest fires protection and prevention. The HOLISTIC Project has been finally accepted in 2013 as Adriatic IPA project under priority 2 Natural and Cultural Resources and Risk Prevention, measure 2.2 Natural and cultural resource management and prevention of natural and technological risks. HOLISTIC project, with full name Adriatic Holistic Forest Fire Protection, ended in December 2016 (Stipanicev et al, 2018). A part of this project was to establish user friendly and simple regional wildfire propagation and wildfire risk prediction simulation tool that could be easily used by fire fighters in everyday fire fighting operative work, but also as a tool for fire season preparation. The final results were AdriaFirePropagator and AdriaFireRisk and additionally AdriaFireRisk sub-module named AdriaFireRiskPanels. These three operative tools are the main topics of this paper. 


\section{Simulator description and architecture}

Wildfires can have severe consequences to the environment, therefore many efforts have been put into investigating events related to fire ignition and fire spread. Several models have been developed throughout the years: "fire ignition modelling", "fire intensity modelling" and "fire spread modelling". "Fire spread modelling" is useful not only during the actual wildfire for predicting how wildfires will spread, but also in pre- and post-fire activities. In the pre-fire, preventive phase, it can be used for organizing the fire fighting units, planning the position of ground fire fighting equipment, or simply for training the fire fighting personnel. In the case of post-fire activities, previous wildfires can be investigated to improve efficiency in the future.

Along with development of fire spread models, fire risk models have improved as well. Fire risk index is a numerical indicator that describes the level of fire risk at certain geographical area. Identifying the fire danger is important for both wildfire prevention and protection. It can be useful for improving automatic smoke and fire detection systems, as well as for other fire-fighting activities that happen before the actual wildfire.

Both fire spread models and fire risk models strongly rely on correct input parameters. Speaking of fire propagation, most of the existing models that have been developed in the last 50 years are mostly based on the following parameters: meteorological conditions (wind speed and direction, temperature, humidity), vegetation (fuel) models, fuel moisture and topography, primarily aspect and slope of the terrain. Similarly, most of the existing fire risk models rely on the very same parameters, although they often consider at least a few other parameters as well. Therefore, a system with a robust and timely access to current meteorological conditions along with an up-to-date GIS database is necessary to develop either a fire spread simulation or fire risk calculation system.

Fire spread systems are usually grouped into three categories:

- Physical and quasi-physical models,

- Empirical and quasi-empirical models,

- Models based on mathematical analogies and simulation models.

Physical models are based on physical and chemical laws related to fire propagation, while quasiphysical models are based solely on physical description of fire propagation. Empirical models are predicting more probable fire behaviour from average conditions and accumulating acknowledges obtained from laboratory and outdoor experimental fire, or historical fires. Semi-empirical models combine analytic formulation of physical phenomena with statistical information. Models based on mathematical analogies use mathematical laws instead of physical, whereas simulation models use any of the aforementioned models with the main aim of simulation of fire spread in two-dimensional space. One of the most famous fire spread models is Rothermel's surface fire spread model. It is a semiphysical model, meaning that it has components that rely on the physics of combustion and components that rely on laboratory experimental results.

The overall system architecture of AdriaFirePropagator is based on two basic modules:

- "Before wildfire" module that includes fire spread simulation based on archived meteorological data (education and investigation mode suitable for pre-fire and post-fire activities), and

- "During wildfire" module that includes fire spread simulation based on real-time meteorological data

AdriaFirePropagator has two main data sources that ensure the correct operation of the system. First, and most important one is a GIS database. GIS database stores not only standard GIS data (such as terrain map, satellite map, road map, settlements, water sources...), but also GIS data specific for 
both fire spread simulation and fire risk index calculation systems. Some of GIS layers are used solely for visualisation and are not required for actual calculations, however, without those layers it would be difficult to visualize the simulation results to the client.

The second data source is a meteorological database. Meteorological data is downloaded automatically several times a day from official server of Croatian Meteorological and Hydrological Service (DHMZ, 2018), with hourly forecast based on ALADIN model, allowing the "during wildfire" module to run simulations throughout the day. Simultaneously, meteorological data is stored in the database for future use when "before wildfire" module is executed.

Fire propagation module is responsible for the actual wildfire simulation and provides simulation results. It is initiated once the user starts a simulation from a web-based user interface. If all input data is provided, the fire propagation module produces simulation results that are then displayed back to the user in the same web-based user interface.

The main tool behind the fire propagation module is a Geographical Information System (GIS), more specifically GRASS GIS, a free, open source system capable of handling raster, topological vector, image processing, and graphic data. We have improved the original fire spread module, developed for GRASS GIS, based on Rothermel's surface fire spread model and cellular automata approach, to allow more customisation of all parameters, with special emphasis on customisation of fuel parameters. This allowed us to better adapt the fire simulation module to Mediterranean climate and vegetation.

Along with fire propagation module, the same GIS is used for two additional modules responsible for calculation of AdriaFireRisk - overall wildfire risk index and AdriaFireEruptiveRisk - eruptive wildfire risk index. AdriaFireRisk module is used to calculate fire risk that is adapted to the microlocation (site-specific wildfire risk index), while AdriaFireEruptiveRisk module is used to detect zones where eruptive fire behaviour could happen.

Both risk indexes are calculated automatically in the background every three hours. Resulting risk maps are served through a WMS service and displayed as layers within AdriaFirePropagator system.

AdriaFirePropagator is a Web based application so users can access the system from any location, using any available wired or wireless ways of accessing the Internet. It is also designed as a multi-user environment, meaning that multiple users can use the system simultaneously and independently. After authenticating with the system, all users are presented with their own user interface that they can customize to their needs and from which they control all their simulation actions, independently of other users.

An additional API was also developed that allows other (authorized) systems to communicate with AdriaFirePropagator. API calls can easily be used to initiate fire propagation module, either in "before wildfire" or "during wildfire" mode, but also to obtain the results of such simulations. Similarly, AdriaFirePropagator allows other systems to display results of AdriaFireRisk and AdriaFireEruptiveRisk calculations using WMS services. This allows other systems to use AdriaFirePropagator as a "single-click" operation to simplify usage in case of emergency. However, it also uses a high degree of customization for more advanced users.

\section{AdriaFirePropagator}

AdriaFirePropagator is based on our previous experience with experimental wildfire spread simulation for Split-Dalmatia County and the following:

- Generic model of Web based wildfire behaviour modelling and wildfire spread simulation adapted for Adriatic region characteristics in HOLISTIC project,

- $\quad$ MOPP - experimental system for wildfire spread simulation developed at University of Split for Split-Dalmatia County (CIPOP, 2015), 
- Wildfire spread simulation modules of Open Source GIS system GRASS GISS developed by various authors under MIT license (GRASS GIS ROS, 2015), but further modified through MOPP and HOLISTIC project.

AdriaFirePropagator user interface is shown in Figure 1, together with an example of a typical wildfire simulation result in AdriaFirePropagator. AdriaFirePropagator was designed primarily as a fire fighters software tool that could be used in pre-fire or post-fire activities, as well as during-fire activities. To support this, three main groups of users were introduced:

1. System administrator,

2. Fire-fighting activities planner, and

3. Sentry in the fire-fighting centre.

System administrator has a full access to all the programs, modules and data in the system. Fire fighting activities planer is a fire-fighting operator who uses fire propagation module for simulating wildfire behaviour based on custom weather conditions or conditions extracted from historical data. Sentry in the fire-fighting centre is a fire-fighting operator who uses the fire propagation module in a case of a real wildfire. In this case, the simulation is performed on the actual (current) meteorological conditions and data.

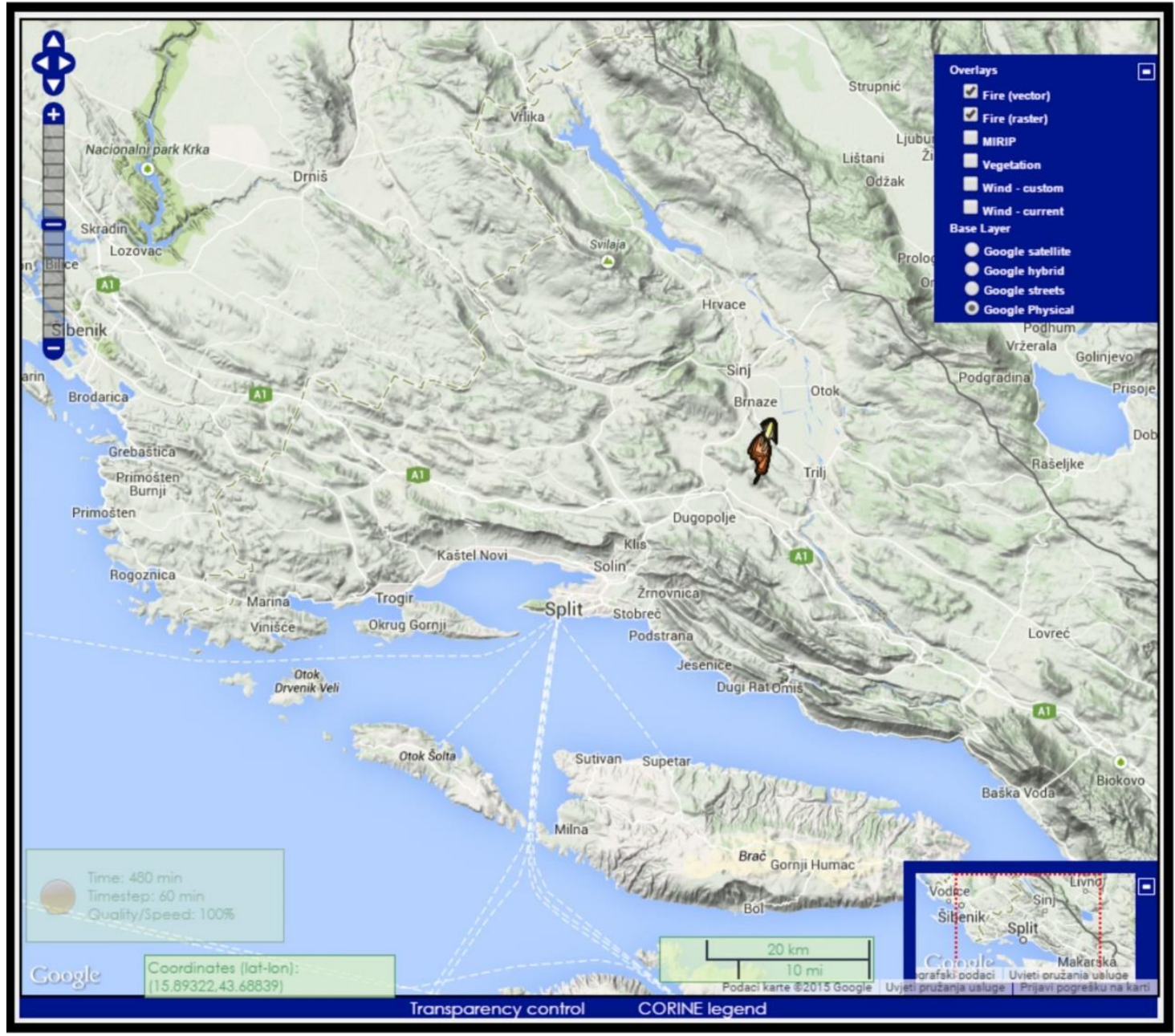

Figure 1 - AdriaFirePropagator screen 
AdriaFirePropagator support two different wildfire simulation scenarios: simulations based on current meteorological conditions and simulations based on custom meteorological conditions, demonstrating two modes of the system: active and simulation mode, respectively.

Before initiating the actual simulation, the user must first specify various simulation settings such as the duration of the simulated fire, time step that will be used to mark the fire front at certain time instants, spatial resolution of the simulation, etc. After choosing the initial point or initial area of the wildfire the user decides whether to run the simulation based on current or custom meteorological conditions. If the user initiates the simulation based on custom meteorological conditions, the system first checks if all the data is prepared. If so, the simulation proceeds with execution. Similarly, if the user initiates the simulation based on current meteorological conditions, the system first checks whether the most recent data is obtained from meteorological server. After preparing all the required data, the simulation proceeds with execution. Finally, all the results are saved in the right format and published in a form of a WMS layer. Fire propagation module then sends a signal to the user interface that automatically refreshes the view to display the latest result.

AdriaFirePropagator checks whether current meteorological data is up to date at the moment when the simulation is run in active mode, other mechanisms exist that do this task on a frequent basis. This is so to avoid unnecessary delay in execution time of the simulation. Time-based job scheduler is set to automatically download meteorological data every three hours from Croatian Hydro Meteorological Service. Current meteorological data is automatically stored into meteorological database (allowing these data for the future use). Simultaneously, all GIS layers that rely on meteorological data are automatically prepared, as explained in the rest of this section. Meteorological data consist of: wind (speed and direction), relative humidity, rainfall and temperature. Most of these data require adjustment before use in propagator. Wind data is first corrected to obtain mid-flame wind speed from standard $10 \mathrm{~m}$ high, whereas relative humidity, rainfall and temperature are used to calculate Canadian Fire Weather Index (FWI) and live, 1-hour, 10-hour and 100-hour fuel moistures. Fuel moisture, both live and dead is a very sensitive parameter in wildfire spread simulation.

Vegetation (fuel) model used in AdriaFirePropagator depends on two inputs: fuel maps and fuel parameters. AdriaFirePropagator supports Albini-Anderson and Scott-Burgan's fuel models as well as fine adjustment of fuel parameters for both, as shown in Figure 2.

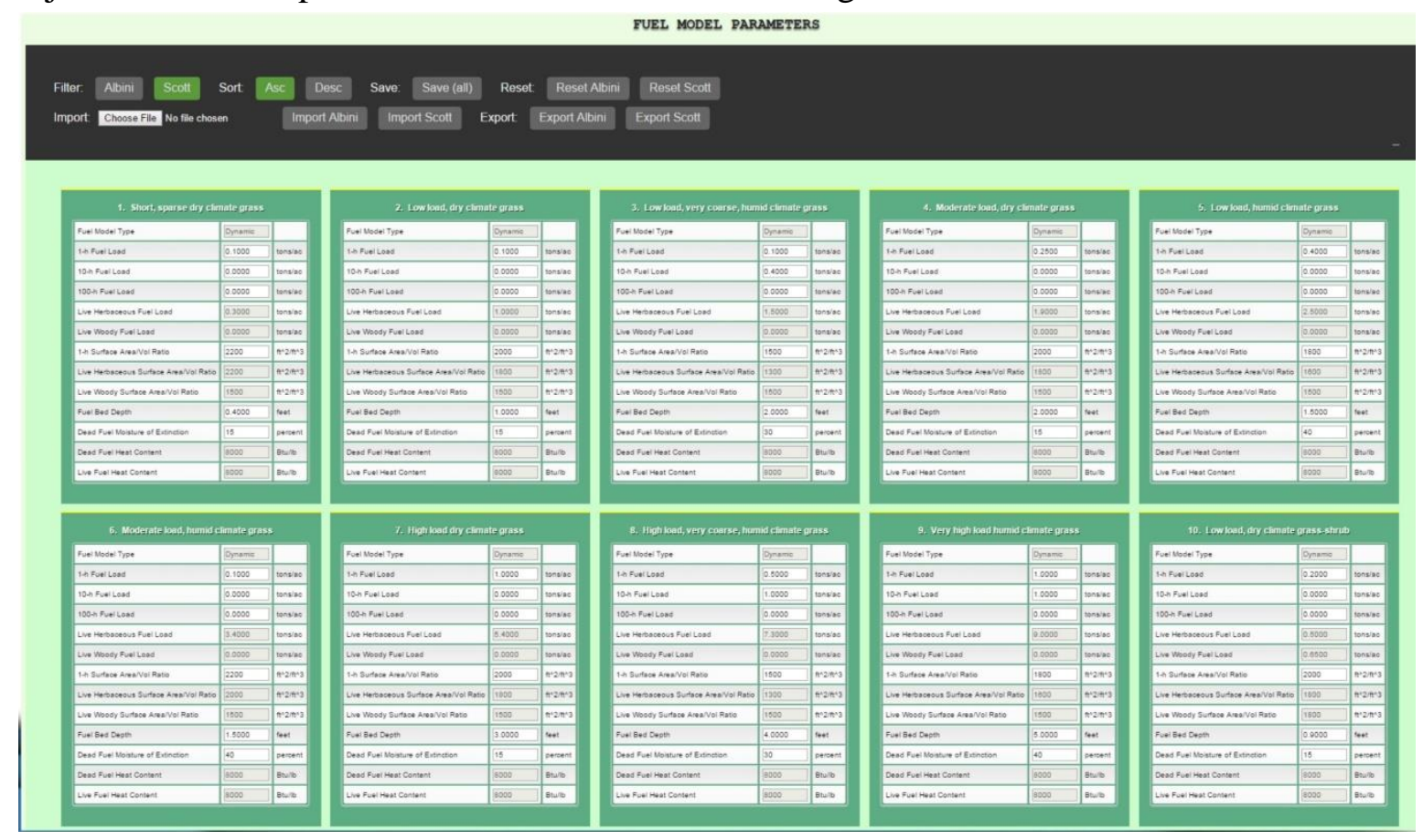

Figure 2 - Adjustment of fuel parameters of applied fuel models. 
Finally, meteorological data, fuel data and topography are used to calculate Rate of spread, a layer that is later used along with ignition point (or area) to calculate the actual simulation result.

Additional possibilities of AdriaFirePropagator is simple adding of fire barriers to simulate unburnable areas and specify the ignition location as a single point or as arbitrary polygon, so that various initial ignition situations could be simulate.

Simultaneously with GIS layers preparation, eruptive wildfire risk index (AdriaFireEruptiveRisk) is calculated. Eruptive fire risk considers the topography of the terrain and current wind data to estimate areas where possible eruptive fire behaviour could happen. Calculations are mostly based on Viegas recommendations (Viegas, 2005). Eruptive wildfire risk is divided in 4 risk categories coded with different shades of red - purple: Category I - Possibility of Landscape caused eruptive fire, Category II - Possibility od Wind \& Landscape caused eruptive fire, Category III - High possibility od Wind \& Landscape caused eruptive fire and Category IV - Very high possibility od Wind \& Landscape caused eruptive fire. Figure 3 shows a typical map where eruptive fire behaviour could happen for part of Split - Dalmatia County on June 30, 2015.

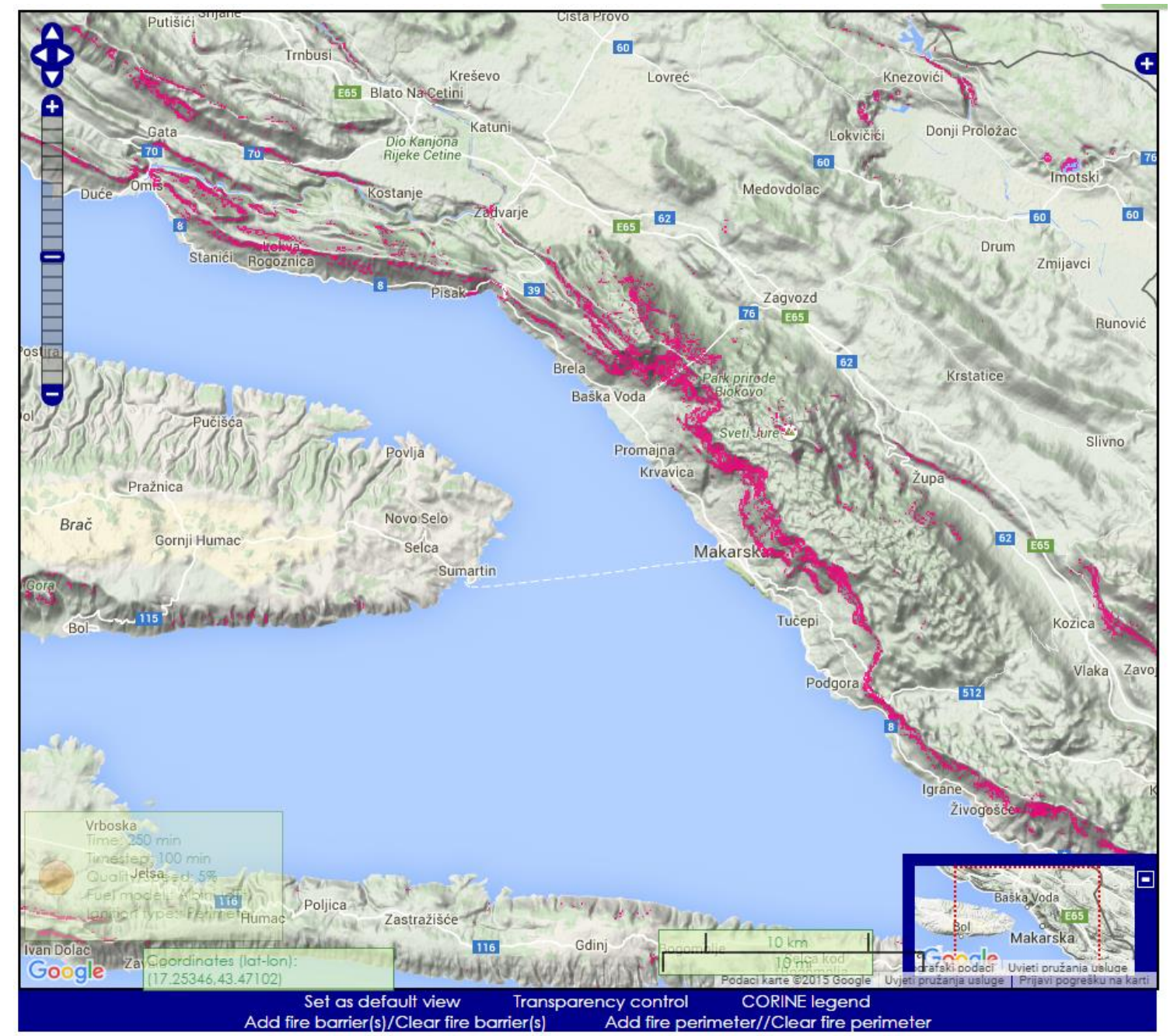

Figure 3 - Map of AdriaFireEruptiveRisk for Split - Dalmatia Counties on June 30, 2015

\section{AdriaFireRisk and AdriaFireRiskPanels}

AdriaFireRisk is an additional module, running parallel on the same server as AdriaFirePropagator, using the same GIS engine based on GRASS GIS application. AdriaFireRisk module calculates sitespecific wildfire risk index based on MIRIP - experimental system for micro location wildfire risk 
index calculation developed at University of Split for Split - Dalmatia County (CIPOP, 2015), (Bugarić et al., 2014).

AdriaFireRisk index is Site-specific Wildfire Risk Index (SWRI) with quite satisfactory spatial resolution. It is not based solely on meteorological parameters, like most existing wildfire risk indexes. It also takes into account other parameters for which we have, after carrying out a detailed statistical analysis, proven that they have a significant influence on the risk of wildfires in the Adriatic region, particularly central part of Adriatic (Bugarić et al., 2014). More specifically, AdriaFireRisk index is based on the following parameters: climatological and meteorological parameters, vegetation, terrain configuration and anthropogenic parameters.

AdriaFireRisk index could be seen as an additional layer of AdriaFirePropagator, but it could be included in any GIS interface as an external WMS layer. An example of the AdriaFireRisk index map for part of Split and Dalmatia County in Croatia, during a small and high-risk index is shown in Fig. 4.
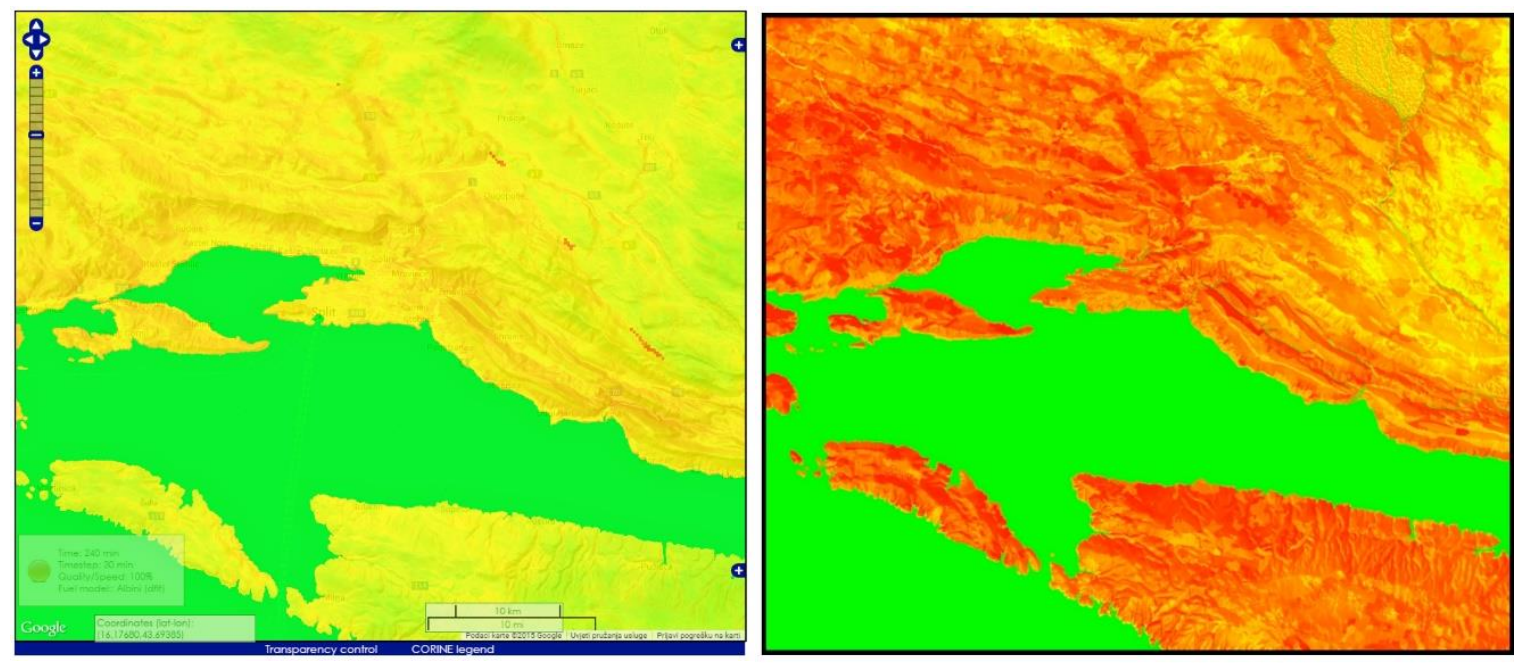

Figure 4 - Site-specific Wildfire Risk Index for part of Split-Dalmatia County during low and high risk.

Climatological and meteorological parameters are dynamic parameters, based on actual meteorological measurements, as well as on prediction of meteorological data calculated by ALADIN numerical weather prediction system of Croatian Meteorological and Hydrological Service (DHMZ). Meteorological data are automatically collected from DHMZ server.

The most important climatological and meteorological parameter within AdriaFireRisk is Canadian Fire Weather Index (FWI). Throughout the years, it has proven to be a good indicator of fire risk, however, it is not well adapted to the micro-location. AdriaFireRisk system calculates FWI every day based on temperature, relative humidity, rainfall and wind data. Meteorological data is also used in combination with other GIS layers. Our study has shown that the slope of the terrain in combination with wind speed or aspect of the terrain in combination with wind direction have greater influence on wildfire risk than wind speed and wind direction alone.

Vegetation is one of the most important parameters that build up fire risk index. FBPS (Fire Behaviour Prediction System) is a fuel model that is used to describe burning characteristics of different vegetation categories. Unfortunately, there is no map that classifies Croatian territory into these fuel categories based on type of vegetation, therefore we decided to use Corine Land Cover map. We have created conversion guidelines that can be used to convert from CLC model to FBPS model.

As already mentioned, topography is used together with meteorological data as an input for AdriaFireRisk calculation. However, terrain configuration has even a more significant influence on fire risk. With higher altitudes the temperatures are lower. Also, there is a direct relation between vegetation types and altitude. 
Finally, proximity to people and infrastructure is of great importance when talking about wildfire risk. Fire risk is higher in the locations inhabited with more people. Historic wildfires have shown high correlation to anthropogenic parameters, more specifically distance to buildings, distance to roads and distance to transmission lines. Therefore, those were considered as additional input for AdriaFireRisk.

AdriaFireRisk is also a support system for additional HOLISTIC project result AdriaFireRiskPanels designed to raise the awareness about the wildfire risk in order to increase caution and reduce the number of accidental wildfires. The panels have been placed on spots of important public interest sensitive to wildfires, such as national parks, city forests, protected islands etc.

Appropriate information about current fire risk for a given area plays an important role in fire prevention. AdriaFireRiskPanels serve as an instrument for disseminating this information to the general public. The main role of these panels is to raise the awareness about wildfires and subsequently influence the behaviour of people in the high-risk areas.

The panels are a visual tool for spreading this information, so they should be easy to interpret, and also legible from larger distances. The panel is divided into five areas of different colours, each representing a certain fire risk index. Figure 5 shows one typical installation at the entrance of protected Park Forest Marjan in Split, Croatia.

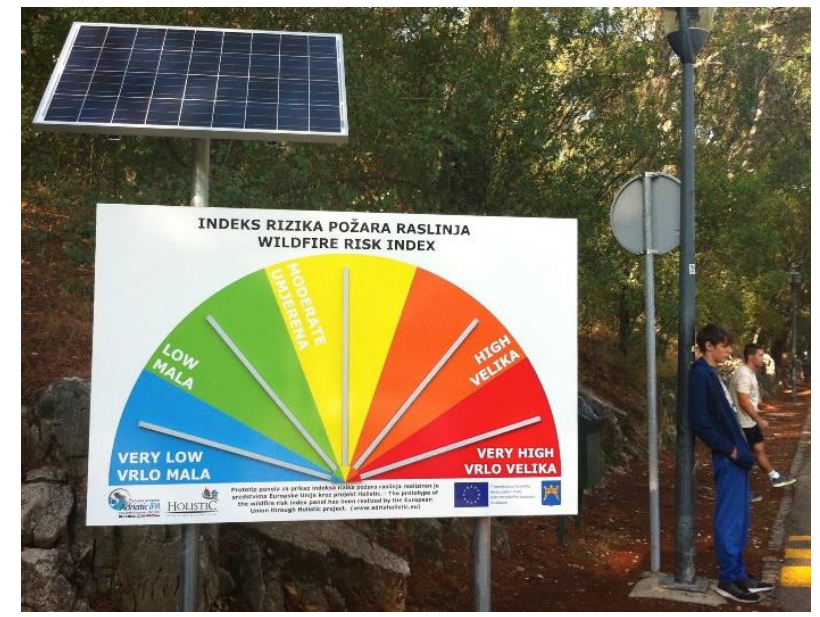

Figure 5 - Wildfire risk index panel installed in Park Forest Marjan in Split, Croatia

LED (Light Emitting Diode) strips are used to signal which fire risk index is currently predicted for the given location. This is indicating by blinking of the strip. Blinking lights are better at drawing attention of the passing by pedestrians than continuous light, and additionally serves as energy conservation mechanism. It is important that the lights are visible even in the midday sunlight, so in the design phase testing was performed to determine which kind of stripes regarding LED type and power categories are most suitable for this kind of application. Figure 5 show the setup used for testing visibility of different LED stripes on different background colours.

Many of potential installation location for fire risk panels are in natural environment, often without access to electrical power source; therefore it is important that these panels are self-sufficient in terms of energy needed for their operation. In such cases panels are equipped with solar panels and deep cycle batteries.

The fire risk index for a given location is calculated on fire risk server and the task of the panel is to retrieve this information and display it properly. The communication between panels and the server is performed using an embedded networking device that has an integrated GPRS modem. This means that the panels must be in range of cellular networks in order to have a means of communication with the server.

The information about the fire index for a given location is served by the server in the form of an XML document that is parsed by the embedded device in the panel. 
The embedded device also operates the relays controlling the LED stripes. It is important that the system is robust when it comes to dealing with communication problems with the servers. The risk index on the panel is refreshed every hour with the information from the server. In case the panel cannot obtain fresh information from the server in the period of 24 hours, the LED stripes are automatically switch off until communication is re-established.

One central server calculates fire risk index for all the locations where panels are installed. Panels do not communicate between themselves, but only with the central server.

Several panels can be deployed for different points in the same location and can use the same fire risk calculation, but generally the deployment is such that panels are deployed for different location and have separate risk calculations. Figure 6 show a diagram for multiple panel system.

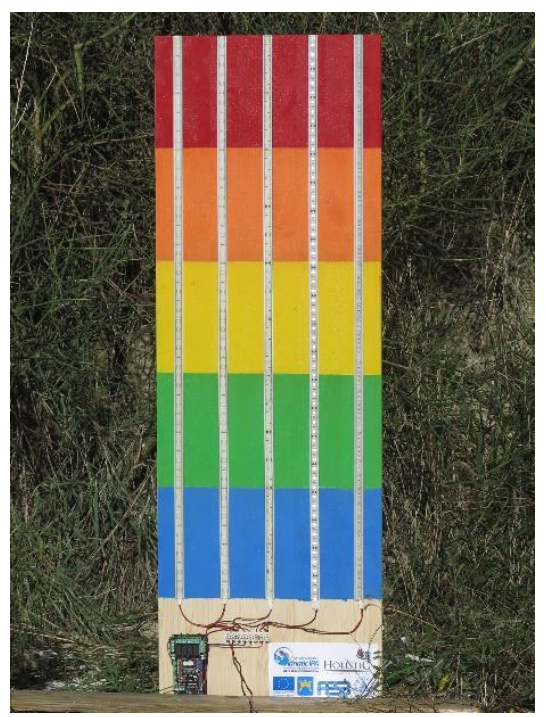

Figure 6 - Setup for LED visibility testing

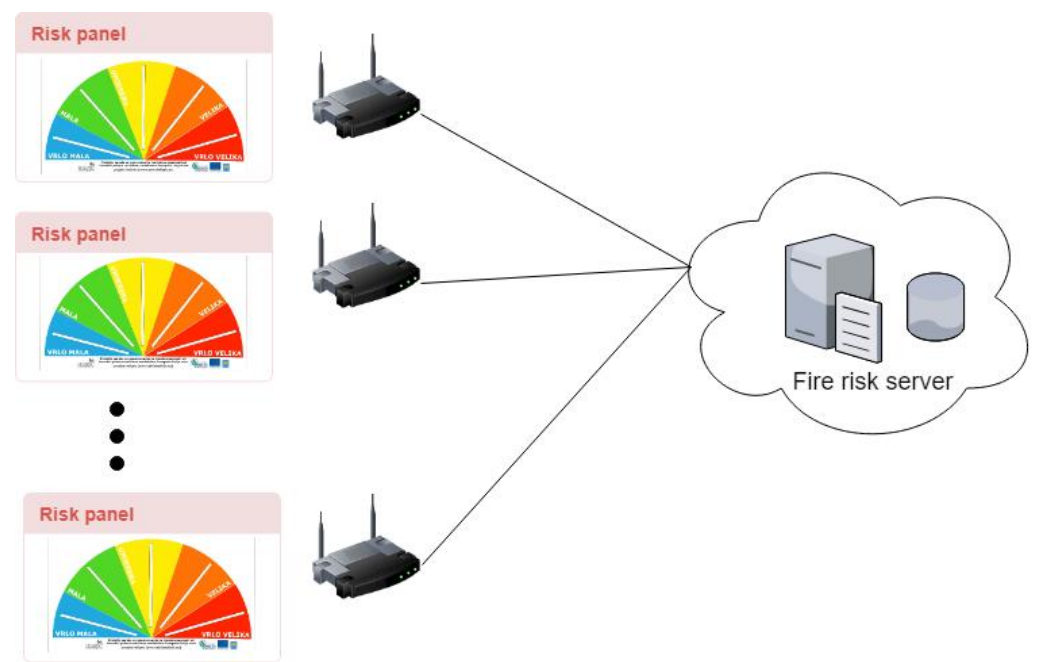

Figure 7 - System with multiple panels

AdriaFireRiskPanels were designed at University of Split Faculty of Electrical Engineering, Mechanical Engineering and Naval Architecture as Open Hardware and Open Software project, so anyone who want to deploy panels could get all project details.

\section{Conclusion}

A part of IPA ADRIATIC HOLISTIC project was to establish user friendly and simple regional wildfire propagation and wildfire risk prediction simulation tool that could be easily used by fire fighters in everyday fire fighting operative work, but also as a tool for fire season preparation. The final results were AdriaFirePropagator and AdriaFireRisk and additionally AdriaFireRisk sub-module named AdriaFireRiskPanels. These three operative tools are the main topics of this paper.

AdriaFirePropagator is a Web based system for wildfire behaviour modelling and wildfire spread simulation. Web based means that all the data are stored on the server, and all the applications in the fire simulation software are server-based programs that run when requested by the users and the only user interface is a standard web browser. 
Based on our previous experience with experimental wildfire spread simulation for Split - Dalmatia County, AdriaFirePropagator was proposed as a software tool for fire fighters that could be used in pre-fire (before-fire) or post-fire (after-fire) activities, as well as for fire (during-fire) activities. In prefire and post-fire activities AdriaFirePropagator could be used for fire behaviour modelling and fire spread simulation based on archived meteorological data (education mode for training and preparing fire fighters for possible future wildfire scenarios, as well as for investigation of some past fires. AdriaFirePropagator could be also a quite useful tool for during-fire activities. In this phase its main task is to predict possible wildfire spread for existing fire, and therefore it is based on real time meteorological data. Knowing a potential fire spread pattern is particularly important for fire fighters safety issues.

AdriaFirePropagator could be used as 'one-click' application, but for more serious fire propagation simulation, a lot of features and parameters can additionally be modified and tuned.

AdriaFireRisk and AdriaFireEruptiveRisk are two additional modules, running in parallel on the same server as AdriaFirePropagator, using the same input data. AdriaFireRisk module calculates sitespecific wildfire risk index. AdriaFireEruptiveRisk module predicts potential areas where there is enhanced risk of eruptive fire behaviour. AdriaFireRisk index is based on several parameters that can be classified into two categories: static and dynamic. Static parameters change slowly over time; therefore they can be computed once before each fire season, while dynamic parameters depend upon current conditions and must be calculated on a daily basis. Those parameters are: climatological and meteorological parameters, vegetation, terrain configuration and anthropogenic parameters. Climatological and meteorological parameters are dynamic parameters, based on actual meteorological measurements, as well as on prediction of meteorological data calculated by ALADIN numerical weather prediction system of Croatian Meteorological and Hydrological Service (DHMZ). Meteorological data are automatically collected from DHMZ server. Other parameters belong to the set of static indicators and they were collected for the all partner participating in HOLISTIC project. AdriaFireRisk index could be seen as an additional layer of AdriaFirePropagator, but it could be included in any GIS interface as an external WMS layer.

AdriaFireRisk is also a support system for additional HOLISTIC project result AdriaFireRiskPanels designed to raise the awareness about the wildfire risk in order to increase caution and reduce the number of accidental wildfires. The panels have been placed on spots of important public interest sensitive to wildfires, such as national parks, city forests, protected islands etc. The paper describes technical details, particularly panels automatic synchronization with AdriaFireRisk system.

\section{References}

Bugarić et al., 2014 Bugarić, M., Braović, M., Stipaničev, D. Statistical evaluation of site-specific wildfire risk index calculation for Adriatic regions // Proceedings of VII International Conference on Forest Fire Research / D.X.Viegas (ur.). Coimbra, Portugal, 17-20.11.2014.

CiPOP, 2015 Center for Wildfire Research, http://cipop.fesb.hr

DHMZ, 2018) Croatian Meteorological and Hydrological Service,

http://www.dhmz.htnet.hr/index_en.php

GRASS GIS ROS, 2015 GRASS GIS SOFTWARE - http://grass.osgeo.org

Stipaničev et al., 2018 Stipaničev, D., Šeric, Lj., Krstinić D., Bugarić, M., Vuković A. "Adriatic Holistic Forest Fire Protection Project - results and experiences a year after" VIII Int. Conference on Forest Fire Research. Coimbra, 2018. (this conference)

Viegas, 2005 Viegas D.X. Forest Fire Meteorology Reaearch and Application, Presentation at WWRP Wildfire Workshop, Melburn, Australia, 6-10 June 2005 